\title{
Presegmentation Procedure Generates Smooth-Sided Microfluidic Devices: Unlocking Multiangle Imaging for Everyone?
}

\author{
Axel Hochstetter*, ${ }^{\dagger}, \ddagger$ (i) \\ ${ }^{\dagger}$ Division of Infection Medicine, Department of Clinical Sciences, Lund University, Lund 221 84, Sweden \\ ${ }^{\ddagger}$ Division of Biomedical Engineering, School of Engineering, University of Glasgow, Glasgow G12 8LT, U.K.
}

\author{
Supporting Information
}

ABSTRACT: We present a simple procedure to create smooth-sided, transparent polymer-based microfluidic devices by presegmentation with hydrophobized glass slides. We study the hypothesis that the smooth side planes permit rapid multiangle imaging of microfluidic systems in contrast to the turbid side planes that result from cutting the polymer. We compare the compatibility of the entire approach with the conventional widefield microscopy, confocal and 2-photon microscopy, as well as three-dimensional (3D) rendering and discuss limitations and potential applications.

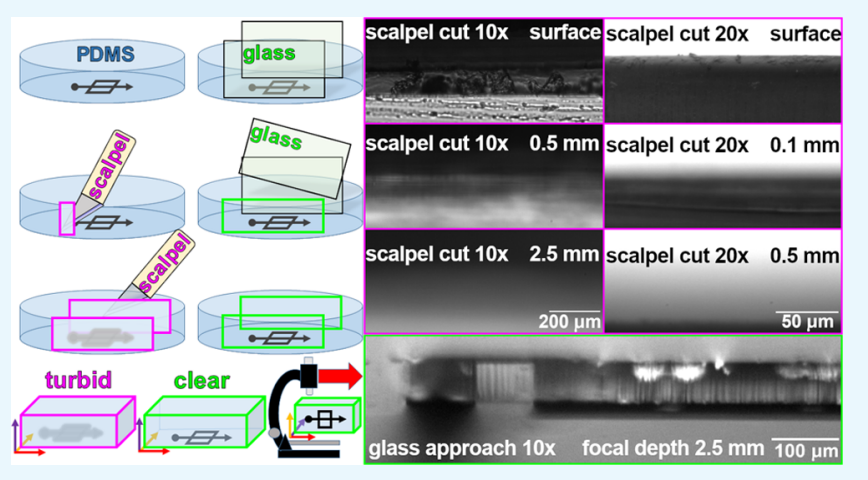

\section{INTRODUCTION}

Poly-dimethylsiloxane (PDMS) is an indispensable tool in today's laboratory-on-a-chip research and microfluidics in general, largely because of its easy molding, biocompatibility, and rapid-prototyping at low cost, and especially its transparency. ${ }^{1-3}$ Often, cast PDMS devices are cut and subsequently bonded to glass or PDMS parts to make microscopecompatible devices for microfluidic studies. ${ }^{4-6}$

While this is a rapid and convenient route, cutting the edges of PDMS generates turbid areas that scatter the light, inhibiting microscopic imaging through this angle. In some applications in biological research, it could be useful to visualize samples from different angles at different stages in the experiment, which is not possible in devices with turbid sides.

The established multiangle microscopic methods to generate two-dimensional (2D) and three-dimensional (3D) reconstructions of samples employ optical coherence tomography, ${ }^{7,8}$ Fourier ptychography of light field measurements, ${ }^{9}$ deconvolution of lens-free images, ${ }^{10}$ or ultrasonography ${ }^{11,12}$ and generally rely heavily on extensive image processing, while not generating 2D images of perpendicular planes. Recently, light field measurement ${ }^{9}$ and polarized multiangle total internal reflection fluorescence (MA-TIRF) achieved live cell imaging below the lateral resolution limit but only at a frequency of one image every $2 \mathrm{~s}^{13}$

In contrast to these methods, which employ only two sides (top and bottom) of their sample for multiangle imaging, we have devised a method that will generate devices with four optically clear sides (top, bottom, front, and back) through which imaging can take place. By using polished, hydrophobized glass slides, inserted into the PDMS prior to curing, we can generate clear sided walls that can be used for rapid multiangle imaging of microfluidic devices across perpendicular planes, e.g., the $x-y$-plane and the $x-z$-plane.

An alternative way to achieve a high spatial and temporal resolution in a thick sample (i.e., the proposed devices) is the usage of light sheet (fluorescence) microscopy (LSM). ${ }^{14}$ There, the sample is not illuminated along the same optical pathway that is used to observe the sample but by a sheet of light that spans the $x-y$-plane but has only minimal thickness in the $z$-direction. ${ }^{14}$ For this illumination, often Bessel beams or Airy beams of monochromatic light or lasers are used, ${ }^{15}$ for they are shape-preserving and self-healing, ${ }^{14,16,17}$ and thus able to illuminate several objects in their path without the loss of accuracy due to diffraction.

\section{RESULTS AND DISCUSSION}

2.1. Widefield Microscopy. Our simple approach enabled us to record images of our microfluidic system from top view, side-on view, and, when shining the light at an angle, an enhanced view that shows more information about the device structure (see Figure 1B-D). The enhancement was caused by reflection at the air-PDMS interface and complete transmission at the PDMS-PDMS interface between the two device halves.

Within the microfluidic system, fluorescently labeled silica beads and internal structures of the microfluidic system (pillars, corners, inlets) were visible in both the $x-y$-plane and the $x-z$-plane, allowing us to determine their position with

Received: July 11, 2019

Accepted: November 14, 2019

Published: December 2, 2019 


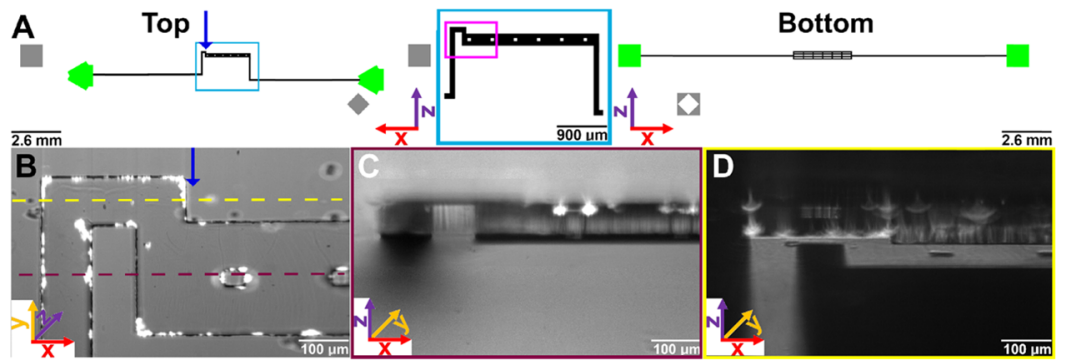

Figure 1. Layout, top-down, side-on, and enhanced views of the microfluidic platform filled with a solution of Rhodamine B coated silica microspheres. (A) Mask layout of the device's top part (left), bottom part, which was kept to a flat surface (right), and a close-up on the imaged section (middle). The inner architecture of the device (black) connects the inlets to the outlets (green). Specific geometries (gray) are used to align the top and bottom parts of the device. Images of the section indicated by the magenta rectangle were taken using brightfield and fluorescence light sources at the same time. The dark blue arrow indicates the optical pathway of C and D. (B) Top-down $(x-y$-plane) view of the indicated section of the device, simultaneously illuminated for fluorescence and brightfield. (C) Side-on view ( $x-z$-plane) into the same device, with the plane of focus indicated by the dark red dashed line and the viewing side indicated by the dark blue arrow. (D) An enhanced view of the same section of the device; the focal plane was slightly shifted compared to $C$, as indicated in yellow, and the source for the brightfield illumination was projected at an angle. Thus, the light was reflected on the PDMS-air interface (gray areas) but transmitted on the PDMS-PDMS interface (black), which resulted in the distinct projection of the pillars (black rectangles within gray areas). Nota bene (NB): B and C are shown in annotated form in Figure 2A, C.

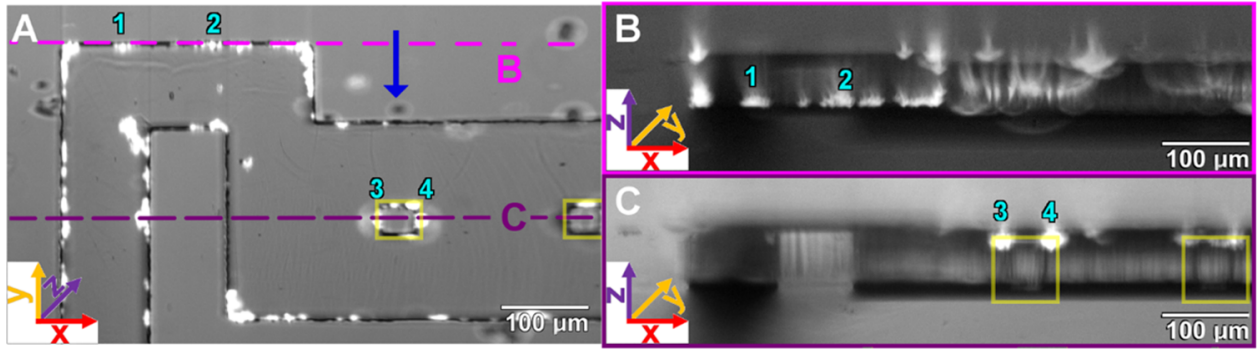

Figure 2. Top-down view and side-on views of the device taken by simultaneous brightfield and fluorescence imaging. (A) Same top-down view of the device as seen in Figure 1B, with the positions of selected beads indicated by numbers and that of pillars by yellow rectangles. The dark blue arrow indicates the optical pathway of B and C. Dashed lines and letters indicate focal planes corresponding to the side-on views in B and C (see matching color frame). (B, C) Side-on views into the device along the planes indicated in A. Yellow rectangles indicate the position of the pillars, and teal colored numbers indicate the corresponding beads in each image. Scale bar is the same for all panels and indicates $100 \mu \mathrm{m}$. NB: for unannotated versions of $\mathrm{A}$ and $\mathrm{C}$, see Figure $1 \mathrm{~B}, \mathrm{C}$.
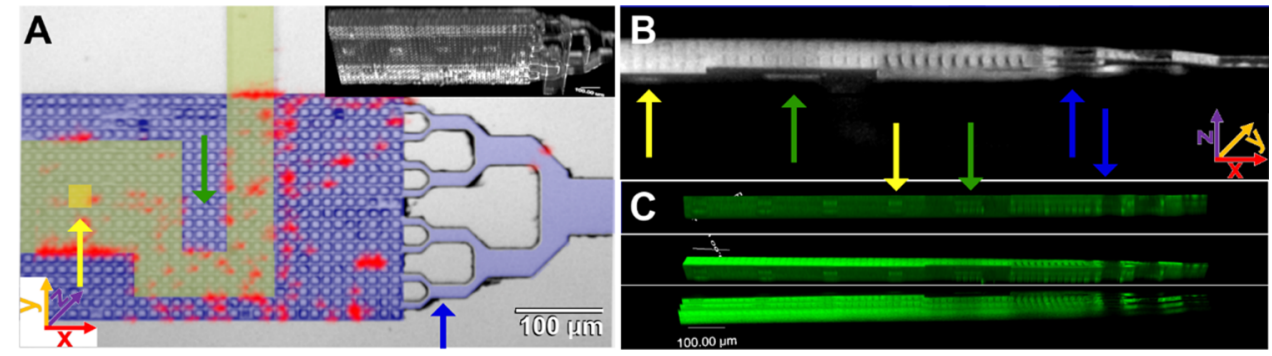

Figure 3. Dual-structure device with arrow markers for pillars (yellow), bottom manifold (blue), and top $z$-bend (green). (A) Top-down widefield micrograph of a dual-structure device with bottom (blue) and top (green) structures, including pillars (yellow). Fluorescent beads have been recorded using 2-photon microscopy (red); their distribution shows that the halves are connected. The inset shows a 3D model rendered from topdown images. (B) Side-on view of the dual structure recorded in widefield with inverted colors and maximized contrast. (C) 3D recreation from (B) and 3 additional images of parallel focal planes.

a high spatial resolution by taking only a single image per plane (see Figure 2).

From the side view, we could distinguish whether the beads were located at the top or the bottom of the device chamber. Additionally, the side view exhibited a very low level of background fluorescence from beads on other focal planes. Using a confocal fluorescence microscope for imaging does further reduce this background.

There is one serious limitation to this approach due to the thickness of the generated microfluidic devices. The width of the device in the $x-y$-plane becomes the height upon rotating to image in the $x-z$-plane. This height has to fit under the microscope and, when scanning through the device, the focal plane has to be moved up through this height. This requires the use of long working distance objectives (LWDOs), and we recommend a maximum working distance of at least $3 \mathrm{~mm}$.

2.2. Confocal Microscopy. Due to the dimensions of the devices, confocal microscopy also required a LWDO, which limited the use of higher magnification objectives. Thus, the resulting images were of a comparable quality than the widefield images (see the Supporting Information). 
2.3. Two-Photon Microscopy. The experimental twophoton microscopy setup permitted the devices to be scanned in both top-down and side-on devices without the need for LWDOs and thus at higher magnification than widefield and confocal imagery. Using third harmonic generation, it is possible to image interfaces, i.e., PDMS/air (comparable to the enhanced view in Figure 1D, see associated content), which can serve as a substitute to brightfield images but still require scanning and are thus much slower to obtain and only work in setups with minimal scattering, which precluded side-on imaging. An additional advantage of 2-photon microscopy is the insensitivity toward contaminations on the sample surface, which allows for easier handling.

2.4. Three-Dimensional Rendering of Images. We rendered two models from the images: one model was created from the top-down images and another was rendered from the side-on images we recorded from an identical dual-structure device. While the image-processing steps were the same, the side-on model looked better when using more images taken with shorter interspacing than the top-down model, but it could not recreate the same level of detail within the device. This, however, can simply be explained with the presence of the grid in the lower structure, as the images in the gridless single-structure devices are much clearer (see Figure 3B,C). The grid has a lattice length of $15 \mu \mathrm{m}$ with an interspacing of 5 $\mu \mathrm{m}$, which acts like an array of slits that create interferences. While the grid reduced the clarity of side-on widefield micrographs, both confocal and 2-photon microscopies are impervious to this drawback.

2.5. Potential Applications. Such a grid can be used as a support structure for in vitro cell culture, supplying seeded cells with a scaffold that supports the growth and differentiation into tissues (e.g., dermal or cerebral endothelial tissue cultures), while the interspacings can carry nutrients to and metabolites from the cells for further analysis. The possibility to record tissues from both top-down and side-on views, in combination with high-speed imaging, could unlock the realtime visualization of barrier function of cell monolayers, such as gut epithelial cells or endothelial cells. It would be useful to be able to visualize a cell barrier from a side-on view to capture conformational changes to the cells during cell transmigration and other barrier disruptions. Being able to view the cells from the top while they are growing would enable a quick visual confirmation that they have formed a confluent layer prior to starting the experiment.

Other applications that could benefit from visualization in a side-on view would be microfluidic-based drug assays, ${ }^{4,6}$ visualization of the glycocalyx and tissues/organs on a chip. The glycocalyx is an important layer of sugars that coat many cells, and its disruption is important in many diseases. ${ }^{18}$ It has historically been difficult to visualize the glycocalyx of cells, ${ }^{19}$ although the thickness of the layer in cultured cells in response to stimuli can be estimated using $z$-stacks of multiple confocal fluorescence microscopy images. ${ }^{20}$ Direct visualization of the glycocalyx or tissues/organs on a chip from the side view could grant a better resolution and therefore a better estimation of their thickness. It would also enable real-time monitoring of their responses to any kind of stimulus.

\section{CONCLUSIONS}

While the hypothesis holds true that the presegmentation procedure generates clear-sided devices that permit microscopy through four instead of two sides, the device dimensions require the use of long working distance objectives, which limits the magnification and resolution that can be achieved. For 2-photon microscopy, this limit is not valid; however, the setup we used did only show fluorescent signals and required long acquisition times for each individual image.

Brightfield imaging is not possible with 2-photon microscopy; however, third harmonic generation can be used as a substitute for nonfluorescing samples, since it shows interfaces between materials with optical density. Third harmonic generation is a scanning method that requires rather long acquisition times per image.

Using stacks of several images taken from parallel focal planes, we could recreate a 3-dimensional impression of the dual-structure device from both angles and compare them (see Figure 3). While the individual 3D model shows similarities to each other and to the normal micrographs taken from both the same and the orthogonal focal plane, there are pronounced differences and the creation involves massive image processing, which allows creation of artefacts and demands careful handling of the sample images to allow automatization and ensure reproducibility.

The main strength of this approach of generating devices is its simplicity and low cost, which makes it easily accessible to groups with limited resources. Additionally, we have shown that with our approach, we can obtain information about the $x-z$-plane of microfluidic devices in only one image and scan along the device in the $y$-direction, providing additional information on the images taken in the $x-y$-plane.

Gridlike structures can cause interferences in the side-on view, and all structures that are not perfectly orthogonal or vertical to the employed optical axis create artefacts, just as is usual in microscopy.

The usage of two instead of one optical axis means that irregularities in the $z$-axis of the device cannot be neglected any more, as can be seen in the appearance of thin vertical structures at higher magnifications in the inner walls of the device, in Figure 2C. These structures most likely stem from the lithographic process and influence the quality of brightfield images along the $x-z$-plane in these areas. These structures would also be expected in other vertical walls made from photoresist, and so glass slides should always be used for generating the clear sides. These distortions would also preclude imaging along long narrow structures such as channels, so care should be taken during the device design step that any area of interest is not shaped in this way (e.g., the leftmost channel in Figure 2C).

While, in general, it is possible to create devices that allow light microscopy along the $x-y$ - and $x-z$-planes, the long working distance that is needed for upright, inverted, and confocal microscopy preclude higher magnifications. While 2photon microscopy can be used to achieve higher magnifications with such a thick sample, it is still very expensive, does not allow for brightfield imaging, and requires long sampling times for each image.

Light sheet microscopy and the presegmentation procedure might be a very powerful combination, providing high magnification at high frame rates, but it would be a costly combination that has not been tested yet.

All in all, this presegmentation procedure really does generate clear-sided devices that are compatible with common microscopy techniques (Table 1) and unlocks multiangle imaging for many potential applications (e.g., artificial cells, 
Table 1. Comparison of Microscopy Techniques for Thick Samples

\begin{tabular}{|c|c|c|c|c|}
\hline $\begin{array}{l}\text { microscopy } \\
\text { technique }\end{array}$ & costs & $\begin{array}{l}\text { recording } \\
\text { speed }\end{array}$ & $\begin{array}{l}\text { image post } \\
\text { processing }\end{array}$ & $\begin{array}{c}\text { thick } \\
\text { samples }\end{array}$ \\
\hline $\begin{array}{l}\text { light (upright/ } \\
\text { inverted) }\end{array}$ & low & high & optional & $\begin{array}{l}\text { with } \\
\text { LWDO }\end{array}$ \\
\hline confocal & medium & scanning & advised & $\begin{array}{l}\text { with } \\
\text { LWDO }\end{array}$ \\
\hline 2-photon & high & scanning & essential & yes \\
\hline light sheet & medium & high & essential & yes \\
\hline
\end{tabular}

tissue engineering, organs on a chip, replacing animal experiments, etc.) and, arguably, for (almost) everybody.

\section{EXPERIMENTAL SECTION}

4.1. Glass Slide Hydrophobization. The hydrophilicity of untreated glass slides can result in low contact angles at the interface of liquid PDMS and glass, generating a meniscus in the PDMS, which introduces unwanted lens effects at the edges of each PDMS device.

This meniscus can be reduced by hydrophobizing the glass surface. To do this, cleaned, polished glass slides (Menzel Gläser, VWR, U.K.) were exposed to a saturated atmosphere of $1 \mathrm{H}, 1 \mathrm{H}, 2 \mathrm{H}, 2 \mathrm{H}$-perfluoro-octyl-trichloro-silane (Sigma, U.K.) at around $1 \mathrm{mbar}$ for $15 \mathrm{~min}$ to $8 \mathrm{~h}$. Excessive silane was washed off with methanol.

4.2. Device Preparation. The devices were produced with standard soft-lithography methods as described previously. ${ }^{21}$ Briefly, SU 8-3050 photoresist (MicroChem) was spin-coated onto commercially available 4-inch silicon wafers according to the manufacturer's directions and exposed to ultraviolet (UV) light through a mask (Compugraphics Jena, Germany) on a mask aligner (MA6; Süss MicroTec, Switzerland) and developed using UV light of $345 \mathrm{~nm}$ wavelength to create masters. PDMS monomer and curing agent (Corning, U.K.) were mixed in a 10:1 ratio and poured over the masters. The devices used in this paper consisted of a simple open chamber with two inlet/outlet channels and a row of support pillars along the middle (see Figure 1A).

Prior to curing the PDMS, hydrophobized glass slides were placed orthogonally to the silicon wafer on either side of the device design. Glass slides were glued to a supportive structure (e.g., pixel pegboards, Junio, Denmark, see Figure 4) such that they could be held orthogonal to the master and parallel to each other. The supporting structures could be made of any other material and were intended to keep the glass slides at the desired distances and orthogonal to the wafer surface. Once the glass slides were placed in the PDMS, it was cured at $80{ }^{\circ} \mathrm{C}$ overnight.

When pouring the PDMS and placing the glass slides, care was taken to ensure that any parts through which imaging would take place were thin enough that the area of interest would lie within the focal range of the microscope.

4.3. Single-Structure Device. For the first experiments, we used a single structure (see Figure 1A “top", Figure 2) as one half of the device that was combined with a plain cube of PDMS to create the devices used for microscopy.

4.4. Dual-Structure Device. By combining two different structures (see Figure 1A "top and bottom", Figure 3) into one device, more complex experiments can be conducted. The dual-structure device has two sets of inlets and outlets to allow both halves to be supplied independently. The interface between the halves can be fitted with a separating membrane or used to grow tissues in vitro, which allows to study diffusion rates and the tissues response to any kind of stimuli, including metabolomics. To support in vitro growth of tissues, a grid with a lattice length of $15 \mu \mathrm{m}$ plus $5 \mu \mathrm{m}$ interspacing was integrated in the bottom half.

4.5. Device Assembly. Once cured, the PDMS was cut along the ends of the glass slides, pulled from the wafer and peeled from the enveloping glass slides. Due to the elasticity of PDMS, this process is solid and reproducible. Careless handling can result in misaligned glass slides or fingerprints or glove marks on the clean surface, which can be removed by either using sticky tape or washing the PDMS with appropriate solvents (e.g., methanol, isopropanol), with optional ultrasonication and recommended drying. To allow fluid into the devices, holes were cut into the upper part of the device using a biopsy puncher (1.5 mm diameter, KIA, Japan). Two PDMS parts were bonded together after surface activation in reactive oxygen plasma. Top and bottom parts should be aligned such that the sides make a flat surface to allow for proper microscopic imaging (see Figure 4C).

4.6. Widefield Microscopy. Rhodamine B coated silica beads (Bang Labs, 7-9 $\mu \mathrm{m}$ diameter) at a concentration of 200 beads/ $\mathrm{mL}$ were injected into the device, where they stuck to the inner surface and remained while injecting air to create a flow-free device for consistent imaging. The beads were excited

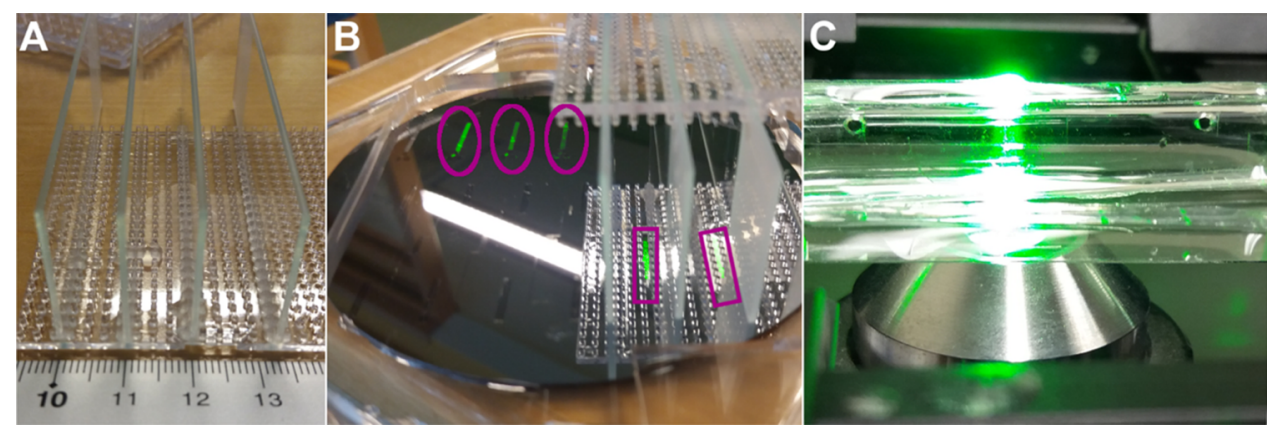

Figure 4. Device preparation: (A) clean microscopy glass slides were hydrophobized and glued onto the supporting scaffolds (e.g., pixel pegboards) at the desired intervals, ensuring that the glass slides were aligned parallel to each other and orthogonal to the supporting scaffolds. (B) After the glass slides were stably affixed to the supporting scaffolds, the resulting construction was placed between the microfluidic structures (tinted in green, with magenta frames for visibility) on top of a master prior to adding and curing the PDMS. (C) Device (consisting of two PDMS halves, covalently bound together) is shown standing upright, with its $x-z$-plane on a glass microscopy slide, while the pathway of light is parallel to the $x-$ $y$-plane of the device. 
with an excitation wavelength of $546 \pm 6 \mathrm{~nm}$ and detected at an emission wavelength of $607 \pm 40 \mathrm{~nm}$ using a Zeiss $20 \mathrm{HE}$ filter-set on a Zeiss Axio Observer.A1 microscope, with a Zeiss 10X objective (NA: 0.3), fitted with an Andor iXon camera (Oxford Instruments, U.K.). Images were processed using Andor Solis X 3238, Irfan view 4.25, and Gnu Image Manipulation Program (GIMP) 2.8.

4.7. Confocal Imaging. Rhodamine B coated silica beads (Bang Labs, $3 \mu \mathrm{m}$ diameter) at a concentration of $(0.001 \% \mathrm{v} /$ v) were injected into the device, where they stuck to the inner surface and remained while the water evaporated over night at room temperature. This created a flow-free device for consistent imaging. The dye coat was excited at a wavelength of $532 \mathrm{~nm}$ and emitted light was detected at wavelengths longer than $550 \mathrm{~nm}$ on a Zeiss LSM 5 Live microscope, with an onboard camera (Zeiss, Germany). Due to the thickness of the device, only long working distance objectives (LWDO) with a maximum working distance of at least $3 \mathrm{~mm}$ have been used.

4.8. Two-Photon Microscopy. Two-photon imaging was carried out on a custom setup, using commercially available parts at a wavelength of $1050 \mathrm{~nm}$ on the same samples as used for confocal imaging. Rhodamine dye imaged by second harmonic generation and the interface between air and PDMS was imaged using third harmonic generation. ${ }^{22}$

4.9. Image Processing for 3D Rendering. To create the 3D models shown in Figure 3, widefield images were recorded as stacks. To balance nonuniform illumination, matrix-masking method $^{23}$ was applied using python. Corrected images were cropped to the area of interest, inverted, and their contrast and brightness was adjusted to show the structure as bright pixels on a black background. The resulting grayscale images were aligned using StackReg plugin ${ }^{24}$ prior to rendering the final 3D models using $3 \mathrm{D}$ viewer plugin in Fiji. ${ }^{25}$

4.10. Additional Considerations. If tubing will be inserted into the devices in downstream applications, the PDMS must be thick enough to properly hold tubing for fluidic transport. Since a thin layer of PDMS may be needed for imaging, the resulting PDMS parts may be too thin to hold the tubing. Therefore, the bottom and top parts can be either individually cast, or an additional PDMS layer can be bonded to one part to provide the necessary thickness.

To properly seal a microfluidic device, it might be necessary to surround it with at least $1 \mathrm{~mm}$ of PDMS on all sides. If a microscopy glass slide with standard thickness $(1.35-1.6 \mathrm{~mm})$ is used, this might limit the choice of objective to LWDO.

For the recording of images along the $x-z$-plane, it is crucial that the setup of the optical path is adjusted, especially the condenser of the brightfield illumination needs adjustment when imaging the device along the $z$-axis, when using inverted or upright microscopes. The usage of two independent optical pathways, one for the $x-y$-plane and another for the $x-z$-plane observations, can counteract the need for constant adjustment.

4.11. Calculation of Spatial Resolution. The resolution in the $x-y$-plane is the same for conventional brightfield microscopy. With our setup, we could resolve 130 individual pixels per $100 \mu \mathrm{m}$, resulting in a $769 \mathrm{~nm}$ pixel pitch. For the $x-z$-plane, resolution was sufficient to separate individual fluorescent beads (7 $\mu \mathrm{m}$ diameter) within the device (see Figure 2).

Taking these values as minimal spatial resolution, our approach has a resulting maximal voxel size of $4.14 \times 10^{-18} \mathrm{~m}^{3}$. Following the equation below, we can calculate the voxel size $V$ using $N=$ number of pixels resolved along a line of $s \mu \mathrm{m}$ for each of the three dimensions $x, y$, and $z$

$$
V=\frac{N_{x}}{s_{x}} \times \frac{N_{y}}{s_{y}} \times \frac{N_{z}}{s_{z}}
$$

Since the resolution in $x$ and $y$ is equal, we can rewrite the equation eq 1 to

$$
V=\left(\frac{100 \mu \mathrm{m}}{130 \text { pixel }}\right)^{2} \times \frac{7 \mu \mathrm{m}}{1 \text { pixel }}=\frac{4.14 \mu \mathrm{m}^{3}}{\text { voxel }}
$$

\section{ASSOCIATED CONTENT}

\section{Supporting Information}

The Supporting Information is available free of charge at https://pubs.acs.org/doi/10.1021/acsomega.9b02139.

Three-dimensional model of the dual-structure device rendered from images taken along the top-down optical pathway (related to Figure 3A)3 (AVI)

Three-dimensional model rendered from images taken along the side-on optical pathway (related to Figure 3C) (AVI)

Stack of images taken in the side-on view (AVI)

Close-up rotation around $x$-axis; annotated (AVI)

\section{AUTHOR INFORMATION}

\section{Corresponding Author}

*E-mail: axel hochstetter@web.de.

ORCID $\odot$

Axel Hochstetter: 0000-0002-2791-2400

\section{Author Contributions}

Axel Hochstetter wrote the manuscript, took all images depicted herein, and has given approval to the final version of the manuscript.

\section{Funding}

The author gratefully acknowledges the funding of the Swiss National Science Foundation (P2BSP2 172033) and Animalfree Research (Switzerland). This project (ID 324) has received funding from the EU-H2020 research and innovation program under the grant agreement No. 654360 having benefited from the access provided by the LundNanoLabs in Sweden within the framework of the NFFA-Europe Transnational Access Activity.

\section{Notes}

The author declares no competing financial interest.

\section{ACKNOWLEDGMENTS}

I thank Jana "Jane" Fisher for her collaboration and Jon Cooper, Pontus Nordenfelt, and the entire LundNanoLabs staff for their support and fruitful discussions.

\section{ABBREVIATIONS}

LWDO, long working distance objective; PDMS, polydimethylsiloxane; UV, ultraviolet; NB, nota bene

\section{REFERENCES}

(1) Tsougeni, K.; Tserepi, A.; Gogolides, E. Photosensitive Poly(Dimethylsiloxane) Materials for Microfluidic Applications. Microelectron. Eng. 2007, 84, 1104-1108. 
(2) Zhou, J.; Ellis, A. V.; Voelcker, N. H. Recent Developments in PDMS Surface Modification for Microfluidic Devices. Electrophoresis 2010, 31, 2-16.

(3) Fujii, T. PDMS-Based Microfluidic Devices for Biomedical Applications. Microelectron. Eng. 2002, 61-62, 907-914.

(4) Hochstetter, A.; Stellamanns, E.; Deshpande, S.; Uppaluri, S.; Engstler, M.; Pfohl, T. Microfluidics-Based Single Cell Analysis Reveals Drug-Dependent Motility Changes in Trypanosomes. Lab Chip 2015, 15, 1961-1968.

(5) Barrett, M. P.; Cooper, J. M.; Regnault, C.; Holm, S. H.; Beech, J. P.; Tegenfeldt, J. O.; Hochstetter, A. Microfluidics-Based Approaches to the Isolation of African Trypanosomes. Pathogens 2017, 6, 47 .

(6) Regnault, C.; Dheeman, D.; Hochstetter, A. Microfluidic Devices for Drug Assays. High-Throughput 2018, 7, 1-13.

(7) Jonas, S.; Bhattacharya, D.; Khokha, M. K.; Choma, M. A. Microfluidic Characterization of Cilia-Driven Fluid Flow Using Optical Coherence Tomography-Based Particle Tracking Velocimetry. Biomed. Opt. Express 2011, 2, 2022.

(8) Wu, C.; Sudheendran, N.; Singh, M.; Larina, I. V.; Dickinson, M. E.; Larin, K. V. Rotational Imaging Optical Coherence Tomography for Full-Body Mouse Embryonic Imaging. J. Biomed. Opt. 2016, 21, No. 026002.

(9) Tian, L.; Waller, L. 3D Intensity and Phase Imaging from Light Field Measurements in an LED Array Microscope. Optica 2015, 2, 104.

(10) Su, T.-W.; Isikman, S. O.; Bishara, W.; Tseng, D.; Erlinger, A.; Ozcan, A. Multi-Angle Lensless Digital Holography for Depth Resolved Imaging on a Chip. Opt. Express 2010, 18, 9690.

(11) Hu, H.; Ye, C.; Wang, X.; Xu, N. Multi-Angle Spatial Compound Imaging in Ultrasonic Immersion Testing Using a Single Transducer. J. Inst. 2018, 13, P07004.

(12) Jespersen, S. K.; Wilhjelm, J. E.; Sillesen, H. Multi-Angle Compound Imaging. Ultrason. Imaging 1998, 20, 81-102.

(13) Zheng, C.; Zhao, G.; Liu, W.; Chen, Y.; Zhang, Z.; Jin, L.; Xu, Y.; Kuang, C.; Liu, X. Three-Dimensional Super-Resolved Live Cell Imaging through Polarized Multi-Angle TIRF. Opt. Lett. 2018, 43, 1423.

(14) Fahrbach, F. O.; Rohrbach, A. A Line Scanned Light-Sheet Microscope with Phase Shaped Self-Reconstructing Beams. Opt. Express 2010, 18, 24229.

(15) Vettenburg, T.; Dalgarno, H. I. C.; Nylk, J.; Coll-Lladó, C.; Ferrier, D. E. K.; Cižmár, T.; Gunn-Moore, F. J.; Dholakia, K. LightSheet Microscopy Using an Airy Beam. Nat. Methods 2014, 11, 541544.

(16) Kaminer, I.; Segev, M.; Christodoulides, D. N. Self-Accelerating Self-Trapped Optical Beams. Phys. Rev. Lett. 2011, 106, No. 213903.

(17) Wong, C. W. Nonspreading Wave Packets. Am. J. Phys. 1996, 64, 792-799.

(18) Schött, U.; Solomon, C.; Fries, D.; Bentzer, P. The Endothelial Glycocalyx and Its Disruption, Protection and Regeneration: A Narrative Review. Scand. J. Trauma Resusc. Emerg. Med. 2016, $24,48$. (19) Reitsma, S.; Slaaf, D. W.; Vink, H.; van Zandvoort, M. A. M. J.; oude Egbrink, M. G. A. The Endothelial Glycocalyx: Composition, Functions, and Visualization. Pflügers Arch. 2007, 454, 345-359.

(20) Zeng, Y.; Ebong, E. E.; Fu, B. M.; Tarbell, J. M. The Structural Stability of the Endothelial Glycocalyx after Enzymatic Removal of Glycosaminoglycans. PLoS One 2012, 7, No. e43168.

(21) Deshpande, S.; Pfohl, T. Hierarchical Self-Assembly of Actin in Micro-Confinements Using Microfluidics. Biomicrofluidics 2012, 6, 34120 .

(22) Squier, J. A.; Müller, M.; Brakenhoff, G. J.; Wilson, K. R. Third Harmonic Generation Microscopy. Opt. Express 1998, 3, 315.

(23) Nordenfelt, P.; Cooper, J. M.; Hochstetter, A. Matrix-Masking to Balance Nonuniform Illumination in Microscopy. Opt. Express 2018, 26, 17279-17288.

(24) Thévenaz, P.; Ruttimann, U. E.; Unser, M. A Pyramid Approach to Subpixel Registration Based on Intensity. IEEE Trans. Image Process 1998, 7, 27-41.
(25) Schindelin, J.; Arganda-Carreras, I.; Frise, E.; Kaynig, V.; Longair, M.; Pietzsch, T.; Preibisch, S.; Rueden, C.; Saalfeld, S.; Schmid, B.; et al. Fiji: An Open-Source Platform for Biological-Image Analysis. Nat. Methods 2012, 9, 676-682. 